\title{
The recursive inverse eigenvalue problem
}

\author{
Marina $\operatorname{Arav}^{1}$ \\ Daniel Hershkowitz ${ }^{1}$ \\ Volker Mehrmann ${ }^{2} 4$ \\ Hans Schneider ${ }^{3} 4$
}

${ }^{1}$ Department of Mathematics, Technion, Haifa 32000, Israel

${ }^{2}$ Fakultät für Mathematik, TU Chemnitz, D-09107 Chemnitz, FRG.

${ }^{3}$ Mathematics Department, University of Wisconsin, Madison, WI 53706, USA.

${ }^{4}$ Partial support received through Sonderforschungsbereich 393 Numerische Simulation auf massive parallelen Rechnernat TU Chemnitz. 


\begin{abstract}
The recursive inverse eigenvalue problem for matrices is studied, where for each leading principle submatrix an eigenvalue and associated left and right eigenvectors are assigned. Existence and uniqueness results as well as explicit formulas are proven, and applications to nonnegative matrices, $Z$-matrices, $M$-matrices, symmetric matrices, Stieltjes matrices and inverse $M$-matrices are considered.
\end{abstract}




\section{Introduction}

Inverse eigenvalue problems are a very important subclass of inverse problems that arise in the context of mathematical modeling and parameter identification. They have been studied extensively in the last 20 years, see e.g. $[3,5,6,8,12,13,14]$ and the references therein. In particular, the inverse eigenvalue problem for nonnegative matrices is still a topic of very active research, since a necessary and sufficient condition for the existence of a nonnegative matrix with a prescribed spectrum is still an open problem, see $[4,12]$.

In this paper we study inverse eigenvalue problems in a recursive matter, that allows to extend already constructed solutions.

We investigate the following recursive inverse eigenvalue problem of order $n:$

Let $F$ be a field, let $s_{1}, \ldots, s_{n} \in F$ and let

$$
\begin{aligned}
& l_{1}=\left[l_{1,1}\right], l_{2}=\left[\begin{array}{l}
l_{2,1} \\
l_{2,2}
\end{array}\right], \ldots, l_{n}=\left[\begin{array}{c}
l_{n, 1} \\
\vdots \\
l_{n, n}
\end{array}\right], \\
& r_{1}=\left[r_{1,1}\right], r_{2}=\left[\begin{array}{c}
r_{1,2} \\
r_{2,2}
\end{array}\right], \ldots, r_{n}=\left[\begin{array}{c}
r_{1, n} \\
\vdots \\
r_{n, n}
\end{array}\right]
\end{aligned}
$$

be vectors with elements in $F$. Construct a matrix $A \in F^{n, n}$ such that

$$
\left\{\begin{array}{l}
l_{i}^{T} A\langle i\rangle=s_{i} l_{i}^{T} \\
A\langle i\rangle r_{i}=s_{i} r_{i}
\end{array} \quad i=1, \ldots, n,\right.
$$

where $A\langle i\rangle$ denotes the $i$-th leading principal submatrix of $A$.

In the sequel we shall use the notation $\operatorname{RIEP}(n)$ for "the recursive inverse eigenvalue problem of order $n$ ".

It should be noted that most of the results that we present below are recursive solutions (hence the name recursive inverse eigenvalue problem), in the sense that the existence and uniqueness conditions are of a recursive 
nature, i.e., once the existence and/or uniqueness of $\operatorname{RIEP}(n-1)$ have been established, the presented conditions describe when the solution of $\operatorname{RIEP}(n)$ exist and is unique. Such results are very useful, in particular when a solution has been computed and lateron new data become available.

To consider a simple example, let us consider a closed Leontief model in economics which is typically described by the action of a nonnegative matrix $T$ with spectral radius 1 on a vector, see e.g., [2]. A nonnegative eigenvector associated with the eigenvalue 1 of the matrix $T$ then is an equilibrium point of the model, [2], and a model that has such a vector is called feasible.

Suppose now that a feasible model with $n-1$ inputs and $n-1$ outputs, i.e., an $(n-1) \times(n-1)$ nonnegative matrix that describes the model and has an equilibrium point, has been constructed.

An immediate question then is whether adding an input and output to the system can again lead to a feasible model with prescribed equilibrium point. This immediately leads to the recursive inverse eigenvalue problem. Our results give necessary and sufficient conditions for several classes of matrices including nonnegative matrices and M-matrices which are the classes of interest in Leontief models and the analysis of Markov chains, see [2].

Existence and uniqueness of nonnegative solutions is therefore one of the major topics of this paper.

In Section 2 we study the existence and uniqueness of solutions for $\operatorname{RIEP}(n)$ in the general case. Our main result gives a recursive characterization of the solution for $\operatorname{RIEP}(n)$. We also obtain a nonrecursive necessary and sufficient condition for unique solvability as well as an explicit formula for the solution in case of uniqueness.

The results of Section 2 are applied in the subsequent sections to special cases. In Section 3 we discuss nonnegative solutions for $\operatorname{RIEP}(n)$ over the field $\mathbb{R}$ of real numbers. We also introduce a nonrecursive sufficient condition for the existence of a nonnegative solution for $\operatorname{RIEP}(n)$. Uniqueness of nonnegative solutions for $\operatorname{RIEP}(n)$ is discussed in Section 4. In Section 5 we study $Z$-matrix and $M$-matrix solutions for $\operatorname{RIEP}(n)$ over $\mathbb{R}$. In Section 6 we consider real symmetric solutions for $\operatorname{RIEP}(n)$ over $\mathbb{R}$. In Section 7 we consider positive semidefinite real symmetric solutions for $\operatorname{RIEP}(n)$ over $\mathbb{R}$. In Section 8 we combine the results of the previous two sections to obtain 
analogous results for Stieltjes matrices. Finally, in Section 9 we investigate inverse $M$-matrix solutions for $\operatorname{RIEP}(n)$. A summary is given in Section 10 .

\section{Existence and uniqueness results}

In this section we study the existence and uniqueness of solutions for $\operatorname{RIEP}(n)$ in the general case. For this purpose we introduce some further notation. For the vectors $l_{i}, r_{i}$ we set

$$
\tilde{l}_{i}=\left[\begin{array}{c}
l_{i, 1} \\
\vdots \\
l_{i, i-1}
\end{array}\right], \quad \tilde{r}_{i}=\left[\begin{array}{c}
r_{1, i} \\
\vdots \\
r_{i-1, i}
\end{array}\right]
$$

The case $n=1$ is easy to verify.

Proposition 1 If $l_{1,1}=r_{1,1}=0$ then every $1 \times 1$ matrix $A$ solves $\operatorname{RIEP}(1)$. If either $l_{1,1} \neq 0$ or $r_{1,1} \neq 0$ then $A=\left[s_{1}\right]$ is the unique solution for $\operatorname{RIEP}(1)$.

For $n \geq 2$ we have the following recursive characterization of the solution for $\operatorname{RIEP}(n)$.

Theorem 2 Let $n \geq 2$. There exists a solution for $\operatorname{RIEP}(n)$ if and only if there exists a solution $B$ for $\operatorname{RIEP}(n-1)$ such that

$$
l_{n, n}=0 \quad \Longrightarrow \quad \tilde{l}_{n}^{T} B=s_{n} \tilde{l}_{n}^{T},
$$

and

$$
r_{n, n}=0 \quad \Longrightarrow \quad B \tilde{r}_{n}=s_{n} \tilde{r}_{n} .
$$

There exists a unique solution for $\operatorname{RIEP}(n)$ if and only if there exists a unique solution for $\operatorname{RIEP}(n-1)$ and $l_{n, n} r_{n, n} \neq 0$.

Proof. Let $A$ be an $n \times n$ matrix. Partition $A$ as

$$
A=\left[\begin{array}{cc}
B & y \\
x^{T} & z
\end{array}\right],
$$


where $B$ is an $(\mathrm{n}-1) \times(\mathrm{n}-1)$ matrix. Clearly, $A$ solves $\operatorname{RIEP}(n)$ if and only if $B$ solves RIEP(n-1) and

$$
\begin{aligned}
\left(s_{n} I_{n-1}-B\right) \tilde{r}_{n} & =r_{n, n} y, \\
\tilde{l}_{n}^{T}\left(s_{n} I_{n-1}-B\right) & =l_{n, n} x^{T}, \\
x^{T} \tilde{r}_{n}+z r_{n, n} & =s_{n} r_{n, n}, \\
\tilde{l}_{n}^{T} y+z l_{n, n} & =s_{n} l_{n, n} .
\end{aligned}
$$

It thus follows that there exists a solution for $\operatorname{RIEP}(n)$ if and only if there exists a solution $B$ for $\operatorname{RIEP}(\mathrm{n}-1)$ such that the equations (4)-(7) (with unknown $x, y$ and $z$ ) are solvable. We now show that these equations are solvable if and only if (1) and (2) hold. Distinguish between four cases:

1. $r_{n, n}=0, l_{n, n} \neq 0$. Here (4) is equivalent to (2), (5) is equivalent to

$$
x^{T}=\frac{\tilde{l}_{n}^{T}\left(s_{n} I_{n-1}-B\right)}{l_{n, n}},
$$

and (6) then follows from (4). For every $y \in F^{n-1}$ we can find $z \in F$ such that (7) holds.

2. $l_{n, n}=0, r_{n, n} \neq 0$. Here (5) is equivalent to (1), (4) is equivalent to

$$
y=\frac{\left(s_{n} I_{n-1}-B\right) \tilde{r}_{n}}{r_{n, n}},
$$

and (7) then follows from (5). For every $x \in F^{n-1}$ we can find $z \in F$ such that (6) holds.

3. $l_{n, n}=r_{n, n}=0$. Here (4) is equivalent to (2) and (5) is equivalent to (1). For any $x \in F^{n-1}$ with $x^{T} \tilde{r}_{n}=0$ we have (6), and for any $y \in F^{n-1}$ with $\tilde{l}_{n}^{T} y=0$ we have (7), where $z$ can be chosen arbitrarily.

4. $l_{n, n} \neq 0, r_{n, n} \neq 0$. Here (4)-(7) have a unique solution, given by (8), (9) and

$$
z=s_{n}-\frac{\tilde{l}_{n}^{T}\left(s_{n} I_{n-1}-B\right) \tilde{r}_{n}}{l_{n, n} r_{n, n}} .
$$


It follows that (4)-(7) are solvable if and only if (1) and (2) hold.

To prove the uniqueness assertion, note that it follows from our proof that if either $l_{n, n}=0$ or $r_{n, n}=0$ then a solution is not unique, since at least one of the vectors $x, y$ and $z$ can be chosen arbitrarily. If both $l_{n, n} \neq 0$ and $r_{n, n} \neq 0$ then every solution $B$ for $\operatorname{RIEP}(\mathrm{n}-1)$ defines a unique solution $A$ for $\operatorname{RIEP}(n)$. The uniqueness claim follows.

This result is recursive and allows to derive a recursive algorithm to compute the solution, but we do not get explicit nonrecursive conditions that characterize the existence of solutions. In order to get a necessary and sufficient condition for unique solvability as well as an explicit formula for the solution in case of uniqueness, we define the $n \times n$ matrix $R_{n}$ to be the matrix whose columns are $r_{1}, \ldots, r_{n}$ with zeros appended at the bottom to obtain $n$-vectors. Similarly, we define the $n \times n$ matrix $L_{n}$ to be the matrix whose rows are $l_{1}, \ldots, l_{n}$ with zeros appended at the right to obtain $n$-vectors. That is, we have

$$
L_{n}=\left[\begin{array}{cccc}
l_{1,1} & & & \\
l_{2,1} & l_{2,2} & & \\
\vdots & & \ddots & \\
l_{n, 1} & \cdots & l_{n, n-1} & l_{n, n}
\end{array}\right], \quad R_{n}=\left[\begin{array}{cccc}
r_{1,1} & r_{1,2} & \cdots & r_{1, n} \\
& r_{2,2} & & \vdots \\
& & \ddots & r_{n-1, n} \\
& & & r_{n, n}
\end{array}\right]
$$

We denote

$$
S_{n}=\left[\begin{array}{ccccc}
s_{1} & s_{2} & s_{3} & \cdots & s_{n} \\
s_{2} & s_{2} & s_{3} & \cdots & s_{n} \\
s_{3} & s_{3} & s_{3} & \cdots & s_{n} \\
\vdots & & & & \vdots \\
s_{n} & s_{n} & \cdots & \cdots & s_{n}
\end{array}\right] .
$$

Also, we denote by o the Hadamard (or elementwise) product of matrices.

Proposition 3 A solution A for $\operatorname{RIEP}(n)$ satisfies

$$
L_{n} A R_{n}=S_{n} \circ\left(L_{n} R_{n}\right) .
$$

Proof. We prove our claim by induction on $n$. For $n=1$ the claim follows easily. Assume that the assertion holds for $n<k$ and let $n=k$. Partition $A$ 
as in (3). We have

$$
\begin{gathered}
L_{n} A R_{n}=\left[\begin{array}{cc}
L_{n-1} & 0 \\
\tilde{l}_{n}^{T} & l_{n, n}
\end{array}\right]\left[\begin{array}{cc}
B & y \\
x^{T} & z
\end{array}\right]\left[\begin{array}{cc}
R_{n-1} & \tilde{r}_{n} \\
0 & r_{n, n}
\end{array}\right] \\
=\left[\begin{array}{c}
L_{n-1} B R_{n-1} \\
L_{n-1}\left(B \tilde{r}_{n}+r_{n, n} y\right) \\
\left(\tilde{l}_{n}^{T} B+l_{n, n} x^{T}\right) R_{n-1} \\
\left(\tilde{l}_{n}^{T} B+l_{n, n} x^{T}\right) \tilde{r}_{n}+\left(\tilde{l}_{n}^{T} y+l_{n, n} z\right) r_{n, n}
\end{array}\right] .
\end{gathered}
$$

By the inductive assumption we have $L_{n-1} B R_{n-1}=S_{n-1} \circ\left(L_{n-1} R_{n-1}\right)$. Also, by (4) we have $B \tilde{r}_{n}+r_{n, n} y=s_{n} \tilde{r}_{n}$, by (5) we have $\tilde{l}_{n}^{T} B+l_{n, n} x^{T}=s_{n} \tilde{l}_{n}^{T}$, and by (7) we have $\tilde{l}_{n}^{T} y+l_{n, n} z=s_{n} l_{n, n}$. It thus follows that

$$
L_{n} A R_{n}=\left[\begin{array}{cc}
S_{n-1} \circ\left(L_{n-1} R_{n-1}\right) & s_{n} L_{n-1} \tilde{r}_{n} \\
s_{n} \tilde{l}_{n}^{T} R_{n-1} & s_{n}\left(\tilde{l}_{n}^{T} \tilde{r}_{n}+l_{n, n} r_{n, n}\right)
\end{array}\right]=S_{n} \circ\left(L_{n} R_{n}\right) .
$$

In general, the converse of Proposition 3 does not hold, that is, a matrix $A$ satisfying (13) does not necessarily form a solution for $\operatorname{RIEP}(n)$, as is demonstrated by Example 5 below.

Theorem 4 There is a unique solution for $\operatorname{RIEP}(n)$ if and only if

$$
l_{1,1} \neq 0 \quad \text { or } \quad r_{1,1} \neq 0
$$

and

$$
l_{i, i} r_{i, i} \neq 0, \quad i=1, \ldots, n .
$$

Furthermore, the unique solution is given by

$$
A=L_{n}^{-1}\left[S_{n} \circ\left(L_{n} R_{n}\right)\right] R_{n}^{-1} .
$$

Proof. The uniqueness claim follows from Proposition 1 and Theorem 2. The fact that the unique solution for $\operatorname{RIEP}(n)$ is given by (14) follows immediately from Proposition 3.

In the case that the solution is not unique, that is, whenever $l_{1,1}=r_{1,1}=0$ or whenever $l_{i, i}$ or $r_{i, i}$ vanish for some $i>1$, the matrices $L_{n}$ and $R_{n}$ defined in (11) are not invertible. Therefore, in this case (14) is invalid. We conclude this section by an example showing that, in general, a revised form of (14), with inverses replaced by generalized inverses, does not provide a solution for $\operatorname{RIEP}(n)$. 
Example 5 Let

$$
s_{1}=1, \quad s_{2}=2, \quad s_{3}=3,
$$

and let

$$
l_{1}=r_{1}=[1], \quad l_{2}=r_{2}=\left[\begin{array}{l}
0 \\
0
\end{array}\right], \quad l_{3}=r_{3}=\left[\begin{array}{l}
0 \\
1 \\
1
\end{array}\right] .
$$

We have

$$
L=\left[\begin{array}{lll}
1 & 0 & 0 \\
0 & 0 & 0 \\
0 & 1 & 1
\end{array}\right], \quad R=L^{T}, \quad S=\left[\begin{array}{lll}
1 & 2 & 3 \\
2 & 2 & 3 \\
3 & 3 & 3
\end{array}\right] .
$$

Let $L^{+}$and $R^{+}$be the Moore-Penrose inverses of $L$ and $R$ respectively, see [1]. We have

$$
A=L^{+}[S \circ(L R)] R^{+}=\left[\begin{array}{ccc}
1 & 0 & 0 \\
0 & 1.5 & 1.5 \\
0 & 1.5 & 1.5
\end{array}\right] .
$$

Since $A\langle 2\rangle$ does not have an eigenvalue $2, A$ is not a solution for $\operatorname{RIEP}(3)$. Note that we still have $L_{n} A R_{n}=S_{n} \circ\left(L_{n} R_{n}\right)$.

In this section we have characterized solvability of $\operatorname{RIEP}(n)$ over a general field $F$ in terms of recursive conditions. We have also given a necessary and sufficient condition for unique solvability and an explicit formula for the unique solution. In the following sections we shall discuss the special cases of nonnegative matrices, $Z$-matrices, $M$-matrices, real symmetric matrices, positive semidefinite matrices, Stieltjes matrices and inverse $M$-matrices.

\section{Existence of nonnegative solutions}

In this section we apply the results of Section 2 to nonnegative solutions for $\operatorname{RIEP}(n)$ over the field $\mathbb{R}$ of real numbers. A matrix $A \in \mathbb{R}^{n, n}$ is said to be nonnegative [positive] if all elements of $A$ are nonnegative [positive]. In this case we write $A \geq 0[A>0]$. For matrices $A, B \in \mathbb{R}^{n, n}$ we write $A \geq B$ if $A-B \geq 0$ and $A>B$ if $A-B>0$.

In order to state our results we define a vector over $\mathbb{R}$ to be unisign if its nonzero components have the same sign. 
Theorem 6 Let $n \geq 2$. There exists a nonnegative solution for $\operatorname{RIEP}(n)$ if and only if we have

$$
l_{i} \text { or } r_{i} \text { is a unisign nonzero vector } \Longrightarrow s_{i} \geq 0, \quad i=1, \ldots, n,
$$

and there exists a nonnegative solution $B$ for $\operatorname{RIEP}(n-1)$ satisfying

$$
\begin{gathered}
\left\{\begin{array}{l}
\frac{s_{n} \tilde{r}_{n}}{r_{n, n}} \geq \frac{B \tilde{r}_{n}}{r_{n, n}}, \quad r_{n, n} \neq 0 \\
s_{n} \tilde{r}_{n}=B \tilde{r}_{n}, \quad r_{n, n}=0
\end{array},\right. \\
\left\{\begin{array}{ll}
\frac{i_{n}^{T} s_{n}}{l_{n, n}} \geq \frac{i_{n}^{T} B}{l_{n, n}}, & l_{n, n} \neq 0 \\
\tilde{l}_{n}^{T} s_{n}=\tilde{l}_{n}^{T} B, & l_{n, n}=0
\end{array},\right.
\end{gathered}
$$

and

$$
l_{n, n} r_{n, n} \neq 0 \quad \Longrightarrow \quad s_{n}\left(\frac{\tilde{l}_{n}^{T} \tilde{r}_{n}}{l_{n, n} r_{n, n}}-1\right) \leq \frac{\tilde{l}_{n}^{T} B \tilde{r}_{n}}{l_{n, n} r_{n, n}} .
$$

There exists a positive solution for $\operatorname{RIEP}(n)$ if and only if there exists a positive solution $B$ for $\operatorname{RIEP}(n-1)$ such that (15)-(18) hold with strict inequalities and every nonzero unisign vector $l_{i}$ or $r_{i}$ has no zero components.

Proof. Let $A \in \mathbb{R}^{n, n}$. As in the proof of Theorem 2, partition $A$ as in (3), and so $A$ solves $\operatorname{RIEP}(n)$ if and only if $B$ solves $\operatorname{RIEP}(\mathrm{n}-1)$ and (4)(7) hold. Therefore, if $A$ is a nonnegative solution for $\operatorname{RIEP}(n)$ then we have (16)-(18). Also, it follows from the nonnegativity of $A$ that (15) holds. Conversely, assume that (15) holds and that $B$ forms a nonnegative solution for RIEP(n-1) satisfying (16)-(18). We show that in this case we can find nonnegative solutions $x, y$ and $z$ for (4)-(7). Distinguish between four cases:

1. $r_{n, n}=0, l_{n, n} \neq 0$. Here $x$ is given by (8), $y$ can be chosen arbitrarily, and $z$ should be chosen such that (7) holds. It follows from (17) that $x$ is nonnegative. If $s_{n} \geq 0$ then we choose $y=0$, we have $z=s_{n}$, and so we have a nonnegative solution for (4)-(7). If $s_{n}<0$ then, by (15), $l_{n}$ is not unisign and hence $\frac{\bar{l}_{n}^{T}}{l_{n, n}}$ has at least one negative component. It follows that we can find a positive vector $y$ such that $\frac{i_{n}^{T} y}{l_{n, n}}<s_{n}$. Since by (7) we have $z=s_{n}-\frac{i_{n}^{T} y}{l_{n, n}}$, it follows that $z>0$, and so again we have a nonnegative solution for (4)-(7). 
2. $l_{n, n}=0, r_{n, n} \neq 0$. Here $y$ is given by $(9), x$ can be chosen arbitrarily, and $z$ should be chosen such that (6) holds. The proof follows as in the previous case.

3. $l_{n, n}=r_{n, n}=0$. Here $x$ and $y$ should be chosen such that $x^{T} \tilde{r}_{n}=\tilde{l}_{n}^{T} y=$ 0 and $z$ can be chosen arbitrarily. In order to obtain a nonnegative solution we can choose $x, y$ and $z$ to be zero.

4. $l_{n, n} \neq 0, r_{n, n} \neq 0$. Here $x$ is given by (8), $y$ is given by (9), and $z$ is given by (10). It follows from (17), (16) and (18) that $x, y$ and $z$ are nonnegative.

Assume now that $A$ is a positive solution for $\operatorname{RIEP}(n)$. It is easy to verify that in this case (15)-(18) should hold with strict inequalities. Also, for every nonzero unisign vector $l_{i}\left[r_{i}\right]$, the vector $l_{i}^{T} A\langle i\rangle\left[A\langle i\rangle r_{i}\right]$ has no zero components, implying that $l_{i},\left[r_{i}\right]$ has no zero components. Conversely, assume that (15) holds with a strict inequality, that every nonzero unisign vector $l_{i}$ or $r_{i}$ has no zero components, and that $B$ forms a positive solution for $\operatorname{RIEP}(n-1)$ satisfying (16)-(18) with strict inequalities. We show that in this case we can find positive solutions $x, y$ and $z$ for $(4)-(7)$. Note that in Case 1 above, the vector $x$ now becomes positive. Also, since the inequality in (15) is now strict, we have either $s_{n}>0$, in which case we can choose positive $y$ sufficiently small such that $z$ is positive, or $s_{n} \leq 0$, in which case $y$ can be chosen positive as before and the resulting $z$ is positive. The same arguments hold for Case 2. In Case 4, it follows from the strict inequalities (17)-(18) that $x$, $y$ and $z$ are positive. Finally, in Case 3 , since $l_{n}$ and $r_{n}$ both have at least one zero component, it follows that both vectors are not unisign. Hence, we can find positive $x$ and $y$ such that $x^{T} \tilde{r}_{n}=\tilde{l}_{n}^{T} y=0$. We assign any positive number to $z$ to find a positive solution $A$ for $\operatorname{RIEP}(n)$.

By the Perron-Frobenius theory, see e.g. [9, 2], the largest absolute value $\rho(A)$ of an eigenvalue of a nonnegative $n \times n$ matrix $A$ is itself an eigenvalue of $A$, the so called Perron root of $A$, and it has an associated nonnegative eigenvector. Furthermore, if $A$ is irreducible, that is, if either $n=1$ or $n \geq 2$ and there exists no permutation matrix $P$ such that $P^{T} A P=\left[\begin{array}{cc}B & \bar{C} \\ 0 & D\end{array}\right]$ where $B$ and $D$ are square, then $\rho(A)$ is a simple eigenvalue of $A$ with an associated positive eigenvector. If $A$ is not necessarily irreducible then we have the following, see e.g. [2]. 
Theorem 7 If $B$ is a principal submatrix of a nonnegative square matrix $A$ then $\rho(B) \leq \rho(A)$. Furthermore, $\rho(A)$ is an eigenvalue of some proper principal submatrix of $A$ if and only if $A$ is reducible.

Note that if we require that the $s_{i}$ are the Perron roots of the principal submatrices $A\langle i\rangle, i=1, \ldots, n$, then, by Theorem 7 , we have

$$
0 \leq s_{1} \leq s_{2} \leq \ldots \leq s_{n} .
$$

If, furthermore, all the leading principal submatrices of $A$ are required to be irreducible, then

$$
0 \leq s_{1}<s_{2}<\ldots<s_{n} .
$$

Condition (19) is not sufficient to guarantee that a nonnegative solution $A$ for $\operatorname{RIEP}(n)$ necessarily has $s_{1}, \ldots, s_{n}$ as Perron roots of $A\langle i\rangle, i=1, \ldots, n$, as is demonstrated by the following example.

Example 8 Let

$$
s_{1}=s_{2}=1, \quad s_{3}=2,
$$

and let

$$
l_{1}=r_{1}=[1], \quad l_{2}=r_{2}=\left[\begin{array}{l}
1 \\
0
\end{array}\right], \quad l_{3}=r_{3}=\left[\begin{array}{l}
1 \\
0 \\
1
\end{array}\right] .
$$

The nonnegative matrix

$$
\left[\begin{array}{lll}
1 & 0 & 1 \\
0 & 3 & 0 \\
1 & 0 & 1
\end{array}\right]
$$

solves $\operatorname{RIEP}(3)$. Note that $\rho(A)=3>s_{3}$.

In order to see cases in which $s_{1}, \ldots, s_{n}$ are the Perron roots of $A\langle i\rangle$, $i=1, \ldots, n$, respectively, we prove

Proposition 9 If the vector $l_{n}$ or $r_{n}$ is positive then for a nonnegative solution $A$ for $\operatorname{RIEP}(n)$ we have $\rho(A)=s_{n}$.

Proof. The claim follows immediately from the known fact that a positive eigenvector of a nonnegative matrix corresponds to the spectral radius, see e.g. Theorem 2.1.11 in [2, p. 128]. 
Corollary 10 If for every $i \in\{1, \ldots, n\}$ we have either $l_{i}>0$ or $r_{i}>0$ then for every nonnegative solution $A$ for $\operatorname{RIEP}(n)$ we have $\rho(A\langle i\rangle)=s_{i}$, $i=1, \ldots, n$.

Lemma 11 Assume that there exists a nonnegative solution $A$ for $\operatorname{RIEP}(n)$ such that $\rho(A\langle n-1\rangle)<s_{n}$. If $r_{n} \neq 0$ or $l_{n} \neq 0$ then $\rho(A)=s_{n}$.

Proof. Since $r_{n} \neq 0$ or $l_{n} \neq 0$ it follows that $s_{n}$ is an eigenvalue of $A$. Assume that $s_{n} \neq \rho(A)$. It follows that the nonnegative matrix $A$ has at least two eigenvalues larger than or equal to $s_{n}$. By [7, p. 473], see also [11, Corollary 1], it follows that $\rho(A\langle\mathrm{n}-1\rangle) \geq s_{n}$, which is a contradiction. Therefore, we have $s_{n}=\rho(A)$.

Corollary 12 If for every $i \in\{1, \ldots, n\}$ we have either $r_{i} \neq 0$ or $l_{i} \neq 0$, and if (20) holds then for every nonnegative solution $A$ for $\operatorname{RIEP}(n)$ we have $\rho(A\langle i\rangle)=s_{i}, i=1, \ldots, n$.

Proof. Note that $A\langle 1\rangle)=\left[s_{1}\right]$ and so $\rho(A\langle 1\rangle)=s_{1}$. Our result follows using Lemma 11 repeatedly.

Lemma 13 Assume that $r_{n} \geq 0$ and $r_{n, n} \neq 0$ or that $l_{n} \geq 0$ and $l_{n, n} \neq$ 0 . Then for every nonnegative solution $A$ for $\operatorname{RIEP}(n)$ we have $\rho(A)=$ $\max \left\{\rho(A\langle n-1\rangle), s_{n}\right\}$.

Proof. Without loss of generality, we consider the case where $r_{n} \geq 0$ and $r_{n, n} \neq 0$. If $r_{n}$ is positive then, by Proposition 9, we have $\rho(A)=s_{n}$ and, since by the Perron-Frobenius theory we have $\rho(A\langle\mathrm{n}-1\rangle) \leq \rho(A)$, the result follows. Otherwise, $r_{n}$ has some zero components. Let $\alpha$ be the set of indices $i$ such that $r_{i, n}>0$ and let $\alpha^{c}$ be the complement of $\alpha$ in $\{1, \ldots, n\}$. Note that since $r_{n}$ is a nonnegative eigenvector of the nonnegative matrix $A$ it follows that the submatrix $A\left[\alpha^{c} \mid \alpha\right]$ of $A$, with rows indexed by $\alpha^{c}$ and columns indexed by $\alpha$, is a zero matrix. It follows that $A$ is a reducible matrix and $\rho(A)=\max \left\{\rho\left(A\left[\alpha^{c} \mid \alpha^{c}\right]\right), \rho(A[\alpha \mid \alpha])\right\}$. Note that the subvector $r_{n}[\alpha]$ of $r_{n}$ indexed by $\alpha$ is a positive eigenvector of $A[\alpha \mid \alpha]$ associated with the eigenvalue $s_{n}$. It thus follows that $\rho(A[\alpha \mid \alpha])=s_{n}$. Since $n \in \alpha$ it follows that $A\left[\alpha^{c} \mid \alpha^{c}\right]$ is a submatrix of $A\langle\mathrm{n}-1\rangle$. Thus, by the Perron-Frobenius theory we have $\rho\left(A\left[\alpha^{c} \mid \alpha^{c}\right]\right) \leq \rho(A\langle\mathrm{n}-1\rangle) \leq \rho(A)$. Hence, it follows that $\rho(A)=\max \left\{s_{n-1}, s_{n}\right\}$. 
Corollary 14 Assume that for every $i \in\{1, \ldots, n\}$ we have either $r_{i} \geq 0$ and $r_{i, i} \neq 0$ or $l_{i} \geq 0$ and $l_{i, i} \neq 0$. Then for every nonnegative solution $A$ for $\operatorname{RIEP}(n)$ we have $\rho(A\langle i\rangle)=\max _{j=1, \ldots, i}\left\{s_{j}\right\}$.

Proof. Note that $A\langle 1\rangle)=\left[s_{1}\right]$ and so $\rho(A\langle 1\rangle)=s_{1}$. Our result follows using Lemma 13 repeatedly.

Corollary 15 Assume that for every $i \in\{1, \ldots, n\}$, we have either $r_{i} \geq 0$ and $r_{i, i} \neq 0$ or $l_{i} \geq 0$ and $l_{i, i} \neq 0$. If (19) holds then for every nonnegative solution $A$ we have $\rho(A\langle i\rangle)=s_{i}, i=1, \ldots, n$.

Another interesting consequence of Theorem 4 is the following relationship between the matrix elements and the eigenvectors associated with the Perron roots of the leading principal submatrices of a nonnegative matrix.

Corollary 16 Let $n \geq 2$. Let $A \in \mathbb{R}^{n, n}$ be a nonnegative matrix, let $s_{i}, l_{i}$ and $r_{i}$ be the Perron roots and associated left and right eigenvectors of $A\langle i\rangle$, $i=1, \ldots, n$, respectively, and assume that (20) holds. Let $S_{n}, L_{n}, R_{n}$ be defined as in (11) and (1D). Then

$$
A\langle i\rangle=L_{i}^{-1}\left[S_{i} \circ\left(L_{i} R_{i}\right)\right] R_{i}^{-1}, \quad i=1, \ldots, n .
$$

Proof. Since (20) holds, it follows that $s_{i}$ is not an eigenvalue of $A\langle\mathrm{i}-1\rangle$, $i=2, \ldots, n$. Therefore, it follows from (1) and (2) that $l_{i, i} r_{i, i} \neq 0$. Also, since $l_{1}$ and $r_{1}$ are eigenvectors of $A\langle 1\rangle$, we have $l_{1,1} r_{1,1} \neq 0$. It now follows from Theorem 4 that $A\langle i\rangle$ is the unique solution for $\operatorname{RIEP}(i)$, and is given by $(21)$.

While Theorem 6 provides a recursive characterization for nonnegative solvability of $\operatorname{RIEP}(n)$, in general nonrecursive necessary and sufficient conditions for the existence of nonnegative solution are not known. We now present a nonrecursive sufficient condition.

Corollary 17 Assume that the vectors $l_{i}, r_{i}, i=1, \ldots, n$ are all positive and that the numbers $s_{1}, \ldots, s_{n}$ are all positive. Let

$$
M_{i}^{r}=\max _{j=1, \ldots, i-1} \frac{r_{j, i}}{r_{j, i-1}}, \quad m_{i}^{r}=\min _{j=1, \ldots, i-1} \frac{r_{j, i}}{r_{j, i-1}}
$$




$$
M_{i}^{l}=\max _{j=1, \ldots, i-1} \frac{l_{i, j}}{l_{i-1, j}}, \quad m_{i}^{l}=\min _{j=1, \ldots, i-1} \frac{l_{i, j}}{l_{i-1, j}} .
$$

If we have

$$
\begin{aligned}
s_{i} m_{i}^{r} \geq s_{i-1} M_{i}^{r}, & i=2, \ldots, n, \\
s_{i} m_{i}^{l} \geq s_{i-1} M_{i}^{l}, & i=2, \ldots, n,
\end{aligned}
$$

and

$$
s_{i}\left(\tilde{l}_{i}^{T} \tilde{r}_{i}-l_{i, i} r_{i, i}\right) \leq s_{i-1} \max \left\{m_{i}^{r} \tilde{l}_{i}^{T} r_{i-1}, m_{i}^{l} l_{i-1}^{T} \tilde{r}_{i}\right\}, \quad i=2, \ldots, n,
$$

then there exists a (unique) nonnegative solution $A$ for $\operatorname{RIEP}(n)$.

Furthermore, if all the inequalities (20)-(24) hold with strict inequality then there exists a (unique) positive solution $A$ for $\operatorname{RIEP}(n)$.

Proof. We prove our assertion by induction on $n$. The case $n=1$ is trivial. By the inductive assumption we can find a nonnegative solution $B$ for $\operatorname{RIEP}(n-1)$. Note that

$$
M_{n}^{r} r_{n-1} \geq \tilde{r}_{n} \geq m_{n}^{r} r_{n-1}
$$

Therefore, it follows from (22) that

$$
s_{n} \tilde{r}_{n} \geq s_{n} m_{n}^{r} r_{n-1} \geq s_{n-1} M_{n}^{r} r_{n-1}=M_{n}^{r} B r_{n-1} \geq B \tilde{r}_{n}
$$

and so (16) holds. Similarly we prove that (17) holds. To prove that (18) holds note that by (25) we have $B \tilde{r}_{n} \geq B m_{n}^{r} r_{n-1}=s_{n-1} m_{n}^{r} r_{n-1}$. Similarly, we have $\tilde{l}_{n}^{T} B \geq s_{n-1} m_{n}^{l} l_{n-1}^{T}$. Hence, it follows that $\tilde{l}_{n}^{T} B \tilde{r}_{n} \geq$ $s_{n-1} \max \left\{m_{n}^{r} \tilde{l}_{n}^{T} r_{n-1}, m_{n}^{l} l_{n-1}^{T} \tilde{r}_{n}\right\}$. By (24) applied to $i=n$ we obtain (18). By Theorem 6 , there exists a nonnegative solution for $\operatorname{RIEP}(n)$. The proof of the positive case is similar.

The conditions in Corollary 17 are not necessary as is demonstrated by the following example.

Example 18 Let $s_{1}=1, s_{2}=2, s_{3}=3$ and let

$$
r_{1}=l_{1}=[1], \quad r_{2}=l_{2}=\left[\begin{array}{l}
1 \\
1
\end{array}\right], \quad r_{3}=\left[\begin{array}{l}
3 \\
5 \\
1
\end{array}\right], \quad l_{3}=\left[\begin{array}{l}
2 \\
1 \\
3
\end{array}\right] .
$$


We have $m_{3}^{r}=3, M_{3}^{r}=5, m_{3}^{l}=1$ and $M_{3}^{l}=2$. Note that both (22) and (23) do not hold for $i=3$. Nevertheless, the unique solution for $\operatorname{RIEP}(3)$ is the nonnegative matrix

$$
\left[\begin{array}{lll}
1 & 1 & 1 \\
1 & 1 & 7 \\
1 & 0 & 0
\end{array}\right]
$$

\section{Uniqueness of nonnegative solutions}

When considering uniqueness of nonnegative solutions for $\operatorname{RIEP}(n)$, observe that it is possible that $\operatorname{RIEP}(n)$ does not have a unique solution but does have a unique nonnegative solution, as is demonstrated by the following example.

Example 19 Let

$$
s_{1}=s_{2}=0,
$$

and let

$$
l_{1}=r_{1}=[1], \quad l_{2}=\left[\begin{array}{l}
1 \\
0
\end{array}\right], \quad r_{2}=\left[\begin{array}{l}
1 \\
1
\end{array}\right] .
$$

By Theorem 2, there is no unique solution for $\operatorname{RIEP}(2)$. Indeed, the solutions for $\operatorname{RIEP}(2)$ are all matrices of the form

$$
\left[\begin{array}{cc}
0 & 0 \\
a & -a
\end{array}\right]
$$

Clearly, the zero matrix is the only nonnegative solution for $\operatorname{RIEP}(2)$.

Observe that, unlike in Theorem 2, the existence of a unique nonnegative solution for $\operatorname{RIEP}(n)$ does not necessarily imply the existence of a unique nonnegative solution for $\operatorname{RIEP}(n-1)$, as is demonstrated by the following example.

Example 20 Let

$$
s_{1}=s_{2}=0, \quad s_{3}=2,
$$

and let

$$
l_{1}=r_{1}=[1], \quad l_{2}=\left[\begin{array}{l}
1 \\
0
\end{array}\right], \quad r_{2}=\left[\begin{array}{c}
1 \\
-1
\end{array}\right], \quad l_{3}=r_{3}=\left[\begin{array}{l}
1 \\
1 \\
1
\end{array}\right]
$$


Observe that all matrices of the form

$$
\left[\begin{array}{ll}
0 & 0 \\
a & a
\end{array}\right]
$$

solve RIEP(2), and hence there is no unique nonnegative solution for $\operatorname{RIEP}(2)$. However, the only nonnegative solution for $\operatorname{RIEP}(3)$ is the matrix

$$
\left[\begin{array}{lll}
0 & 0 & 2 \\
1 & 1 & 0 \\
1 & 1 & 0
\end{array}\right] .
$$

We remark that one can easily produce a similar example with nonnegative vectors $r_{i}$ and $l_{i}, i=1, \ldots, n$.

In order to introduce necessary conditions and sufficient conditions for uniqueness of nonnegative solutions for $\operatorname{RIEP}(n)$ we prove

Lemma 21 Let $n \geq 2$, and assume that $B$ forms a nonnegative solution for $\operatorname{RIEP}(n-1)$ satisfying (15)-(18). Then there exist unique nonnegative vectors $x, y$ and $z$ such that the matrix $\left[\begin{array}{cc}B & y \\ x^{T} & z\end{array}\right]$ solves $\operatorname{RIEP}(n)$ if and only if either $l_{n, n} r_{n, n} \neq 0$, or $s_{n}=0$ and $l_{n}$ is a unisign vector with no zero components, or $s_{n}=0$ and $r_{n}$ is a unisign vector with no zero components.

Proof. We follow the proof of Theorem 6 . Consider the four cases in that proof. In Case 1 , the vector $x$ is uniquely determined and any nonnegative assignment for $y$ is valid as long as $z=s_{n}-\frac{\tilde{l}_{n}^{T} y}{l_{n, n}} \geq 0$. If $s_{n}>0$ then every nonnegative vector $y$ sufficiently small will do. If $s_{n}<0$ then, as is shown in the proof of Theorem 6 , we can find a positive $y$ such that $z>0$, and by continuity arguments there exist infinitely many such vectors $y$. If $s_{n}=0$ then a unique such $y$ exists if and only if there exists a unique nonnegative vector $y$ such that $\frac{\tilde{l}_{n}^{T} y}{l_{n, n}} \leq 0$. Clearly, if $\tilde{l}_{n}$ has a nonpositive component then every vector $y$ whose corresponding component is positive and all other components are zero solves the problem. On the other hand, if $\tilde{l}_{n}>0$, which is equivalent to saying that $l_{n}$ is a unisign vector with no zero components, then the only nonnegative vector $y$ that solves the problem is $y=0$. Similarly, we prove that, in case 2 , a unique nonnegative solution exists if and only if $s_{n}=0$ and $r_{n}$ is a unisign vector with no zero components. We do not have 
uniqueness in Case 3 since then $z$ can be chosen arbitrarily. Finally, there is always uniqueness in Case 4.

Lemma 21 yields sufficient conditions and necessary conditions for uniqueness of nonnegative solutions for $\operatorname{RIEP}(n)$. First, observe that if $s_{n}=0$ and $l_{n}$ is a unisign vector with no zero components, or if $s_{n}=0$ and $r_{n}$ is a unisign vector with no zero components, then the zero matrix is the only nonnegative solution of the problem. A less trivial sufficient condition is the following.

Corollary 22 Let $n \geq 2$, and let $A$ be a nonnegative solution for $\operatorname{RIEP}(n)$. If $A\langle n-1\rangle$ forms a unique nonnegative solution for $\operatorname{RIEP}(n-1)$ and if $l_{n, n} r_{n, n} \neq$ 0 , then $A$ is the unique nonnegative solution for $\operatorname{RIEP}(n)$.

Necessary conditions are given by the following

Corollary 23 Let $n \geq 2$. If there exists a unique nonnegative solution for $\operatorname{RIEP}(n)$ then either $l_{n, n} r_{n, n} \neq 0$, or $s_{n}=0$ and $l_{n}$ is a unisign vector with no zero components, or $s_{n}=0$ and $r_{n}$ is a unisign vector with no zero components.

The condition $l_{n, n} r_{n, n} \neq 0$ is not sufficient for the uniqueness of a nonnegative solution for $\operatorname{RIEP}(n)$, as is shown in the following example.

Example 24 Let

$$
s_{1}=s_{2}=s_{3}=0,
$$

and let

$$
l_{1}=r_{1}=[1], \quad l_{2}=\left[\begin{array}{l}
1 \\
0
\end{array}\right], \quad r_{2}=\left[\begin{array}{c}
1 \\
-1
\end{array}\right], \quad l_{3}=r_{3}=\left[\begin{array}{c}
1 \\
-1 \\
1
\end{array}\right]
$$

Although we have $l_{n, n} r_{n, n} \neq 0$, all matrices of the form

$$
\left[\begin{array}{lll}
0 & 0 & 0 \\
a & a & 0 \\
a & a & 0
\end{array}\right]
$$

solve $\operatorname{RIEP}(3)$, and hence there is no unique nonnegative solution for $\operatorname{RIEP}(3)$. 


\section{The $Z$-matrix and $M$-matrix case}

A real square matrix $A$ is said to be a $Z$-matrix if it has nonpositive offdiagonal elements. Note that $A$ can be written as $A=\alpha I-B$ where $\alpha$ is a real number and $B$ is a nonnegative matrix. If we further have that $\alpha \geq \rho(B)$ then we say that $A$ is an $M$-matrix.

In this section we discuss $Z$-matrix and $M$-matrix solutions for $\operatorname{RIEP}(n)$ over the field $\mathbb{R}$ of real numbers. The proofs of the results are very similar to the proofs of the corresponding results in Sections 3 and 4 and, thus, are omitted in most cases.

Theorem 25 Let $n \geq 2$. There exists a $Z$-matrix solution for $\operatorname{RIEP}(n)$ if and only if there exists a $Z$-matrix solution $B$ for $\operatorname{RIEP}(n-1)$ satisfying

$$
\left\{\begin{array}{l}
\frac{s_{n} \tilde{r}_{n}}{r_{n, n}} \leq \frac{B \tilde{r}_{n}}{r_{n, n}}, \quad r_{n, n} \neq 0 \\
s_{n} \tilde{r}_{n}=B \tilde{r}_{n}, \quad r_{n, n}=0
\end{array},\right.
$$

and

$$
\left\{\begin{array}{l}
\frac{\tilde{l}_{n}^{T} s_{n}}{l_{n, n}} \leq \frac{\tilde{l}_{n}^{T} B}{l_{n, n}}, \quad l_{n, n} \neq 0 \\
\tilde{l}_{n}^{T} s_{n}=\tilde{l}_{n}^{T} B, \quad l_{n, n}=0
\end{array} .\right.
$$

Furthermore, if $l_{n}$ or $r_{n}$ is positive then a Z-matrix solution for $\operatorname{RIEP}(n)$ is an $M$-matrix if and only if $s_{n} \geq 0$.

Proof. The proof of the first part of the theorem is similar to the proof of Theorem 6 , observing that here the vectors $x$ and $y$ are required to be nonnegative and that the sign of $z$ is immaterial. The proof of the second part of the Theorem follows, similarly to Proposition 9, from the known fact that a positive eigenvector of a $Z$-matrix corresponds to the least real eigenvalue.

Theorem 26 Let $n \geq 2$. Let $A \in \mathbb{R}^{n, n}$ be a $Z$-matrix, let $s_{i}, l_{i}$ and $r_{i}$ be the least real eigenvalues and the corresponding left and right eigenvectors of $A\langle i\rangle, i=1, \ldots, n$, respectively, and assume that

$$
s_{1}>s_{2}>\ldots>s_{n}
$$


Let $S_{n}, L_{n}, R_{n}$ be defined as in (11) and (12). Then

$$
A\langle i\rangle=L_{i}^{-1}\left[S_{i} \circ\left(L_{i} R_{i}\right)\right] R_{i}^{-1}, \quad i=1, \ldots, n .
$$

For the numbers $M_{i}^{r}, m_{i}^{r}, M_{i}^{l}$ and $m_{i}^{l}$, defined in Corollary 17 , we have

Theorem 27 Assume that the vectors $l_{i}, r_{i}, i=1, \ldots, n$ are all positive and that the numbers $s_{1}, \ldots, s_{n}$ are all positive. If we have

$$
s_{i} M_{i}^{r} \leq s_{i-1} m_{i}^{r}, \quad i=2, \ldots, n
$$

and

$$
s_{i} M_{i}^{l} \leq s_{i-1} m_{i}^{l}, \quad i=2, \ldots, n,
$$

then there exists a (unique) $M$-matrix solution $A$ for $\operatorname{RIEP}(n)$.

Theorem 28 Let $n \geq 2$, let $A$ be a $Z$-matrix solution for $\operatorname{RIEP}(n)$ and assume that $A\langle n-1\rangle$ forms a unique $Z$-matrix solution for $\operatorname{RIEP}(n-1)$. Then $A$ is the unique $Z$-matrix solution for $\operatorname{RIEP}(n)$ if and only if $l_{n, n} r_{n, n} \neq 0$.

Here too, unlike in Theorem 2, the existence of a unique $Z$-matrix solution for $\operatorname{RIEP}(n)$ does not necessarily imply the existence of a unique $Z$-matrix solution for $\operatorname{RIEP}(n-1)$, as is demonstrated by the following example.

Example 29 Let $s_{1}=s_{2}=s_{3}=0$, and let

$$
l_{1}=r_{1}=[1], \quad l_{2}=\left[\begin{array}{l}
1 \\
0
\end{array}\right], \quad r_{2}=\left[\begin{array}{l}
1 \\
1
\end{array}\right], \quad l_{3}=r_{3}=\left[\begin{array}{l}
1 \\
1 \\
1
\end{array}\right] .
$$

Observe that all matrices of the form

$$
\left[\begin{array}{cc}
0 & 0 \\
a & -a
\end{array}\right]
$$

solve $\operatorname{RIEP}(2)$, and hence there is no unique $Z$-matrix solution for $\operatorname{RIEP}(2)$. However, it is easy to verify that the zero matrix is the only $Z$-matrix solution for $\operatorname{RIEP}(3)$. 


\section{The real symmetric case}

The inverse eigenvalue problem for real symmetric matrices is well studied, see e.g. [3]. In this section we consider symmetric solutions for $\operatorname{RIEP}(n)$ over the field $\mathbb{R}$ of real numbers. We obtain the following consequence of Theorem 2, characterizing the real symmetric case.

Theorem 30 Let $n \geq 2$. There exists a symmetric solution for $\operatorname{RIEP}(n)$ if and only if there exists a symmetric solution $B$ for $\operatorname{RIEP}(n-1)$ such that the implications (1) and (D) hold, and

$$
l_{n, n} r_{n, n} \neq 0 \quad \Longrightarrow \quad\left(s_{n} I_{n-1}-B\right)\left(\frac{\tilde{l}_{n}}{l_{n, n}}-\frac{\tilde{r}_{n}}{r_{n, n}}\right)=0 .
$$

Furthermore, if there exists a unique symmetric solution for $\operatorname{RIEP}(n)$ then $l_{n, n} \neq 0$ or $r_{n, n} \neq 0$.

Proof. Let $A \in \mathbb{R}^{n, n}$. Partition $A$ as in (3), and so $A$ solves $\operatorname{RIEP}(n)$ if and only if $B$ solves RIEP(n-1) and (4)-(7) hold. It was shown in the proof of Theorem 2 that (4)-(7) are solvable if and only if (1) and (2) hold. Therefore, all we have to show that if $B$ is symmetric then we can find solutions $x, y$ and $z$ for (4)-(7) such that $y=x$ if and only if (26) holds. We go along the four cases discussed in Theorem 2. In Case 1, the vector $x$ is uniquely determined and the vector $y$ can be chosen arbitrarily. Therefore, in this case we set $y=x$, and $z$ is then uniquely determined. In Case 2 , the vector $y$ is uniquely determined and the vector $x$ can be chosen arbitrarily. Thus, in this case we set $x=y$, and $z$ is then uniquely determined. In Case 3 , we can choose any $x$ and $y$ as long as $x^{T} \tilde{r}_{n}=0$ and $\tilde{l}_{n}^{T} y=0$. In particular, we can choose $x=y=0$. Furthermore, $z$ can be chosen arbitrarily. Finally, in Case 4 , we have $x=y$ if and only if (26) holds. Note that this is the only case in which, under the requirement that $y=x$, the vectors $x, y$ and $z$ are uniquely determined.

We remark that, unlike in Theorem 2 , the existence of a unique symmetric solution for $\operatorname{RIEP}(n)$ does not necessarily imply the existence of a unique symmetric solution for $\operatorname{RIEP}(n-1)$, as is demonstrated by the following example. 
Example 31 Let

$$
s_{1}=1, \quad s_{2}=2, \quad s_{3}=0,
$$

and let

$$
\begin{gathered}
l_{1}=r_{1}=[1], \quad l_{2}=r_{2}=\left[\begin{array}{l}
1 \\
1
\end{array}\right], \quad l_{3}=r_{3}=\left[\begin{array}{c}
1 \\
-1 \\
0
\end{array}\right], \\
l_{4}=\left[\begin{array}{c}
1 \\
0 \\
0 \\
-1
\end{array}\right], \quad r_{4}=\left[\begin{array}{c}
1 \\
1 \\
-1 \\
-1
\end{array}\right] .
\end{gathered}
$$

It is easy to verify that all symmetric matrices of the form

$$
\left[\begin{array}{lll}
1 & 1 & a \\
1 & 1 & a \\
a & a & b
\end{array}\right], \quad a, b \in \mathbb{R}
$$

solve $\operatorname{RIEP}(3)$, while the unique solution for $\operatorname{RIEP}(4)$ is

$$
\left[\begin{array}{llll}
1 & 1 & 1 & 1 \\
1 & 1 & 1 & 1 \\
1 & 1 & 1 & 1 \\
1 & 1 & 1 & 1
\end{array}\right] .
$$

This example also shows that there may exist a unique solution for $\operatorname{RIEP}(n)$ even if $l_{i, i}=r_{i, i}=0$ for some $i \in 1, \ldots, n$.

Naturally, although not necessarily, one may expect in the symmetric case to have the condition

$$
r_{i}=l_{i}, \quad i=1, \ldots, n .
$$

Indeed, in this case we have the following corollary of Theorems 2 and 30.

Corollary 32 Let $n \geq 2$ and assume that (27) holds. The following are equivalent:

(i) There exists a symmetric solution for $\operatorname{RIEP}(n)$.

(ii) There exists a solution for $\operatorname{RIEP}(n)$.

(iii) There exists a symmetric solution $B$ for $\operatorname{RIEP}(n-1)$ such that (1) holds.

(iv) There exists a solution $B$ for $\operatorname{RIEP}(n-1)$ such that (1) holds. 
Proof. Note that since (27) holds, we always have (26). We now prove the equivalence between the four statements of the theorem.

(i) $\Longrightarrow$ (ii) is trivial.

(ii) $\Longrightarrow$ (iv) by Theorem 2 .

(iv) $\Longrightarrow$ (iii). Since (27) holds, it follows that $\frac{B+B^{T}}{2}$ also solves $\operatorname{RIEP}(\mathrm{n}-1)$.

(iii) $\Longrightarrow($ i). Since $B$ is symmetric and since we have (27), the implications

(1) and (2) are identical. Our claim now follows by Theorem 30.

For uniqueness we have

Theorem 33 Let $n \geq 2$ and assume that (27) holds. The following are equivalent:

(i) There exists a unique symmetric solution for $\operatorname{RIEP}(n)$.

(ii) There exists a unique solution for $\operatorname{RIEP}(n)$.

(iii) We have $l_{i, i} \neq 0, i=1, \ldots, n$.

Proof. In view of (27), the equivalence of (ii) and (iii) follows from Theorem 4 . To see that (i) and (iii) are equivalent note that, by the construction in Theorem 30, for every symmetric solution $B$ for $\operatorname{RIEP}(n-1)$ there exists a solution $A$ for $\operatorname{RIEP}(n)$ such that $A\langle\mathrm{n}-1\rangle=B$. Furthermore, $A$ is uniquely determined if and only if $l_{n, n} \neq 0$. Therefore, it follows that there exists a unique symmetric solution for $\operatorname{RIEP}(n)$ if and only if there exists a unique symmetric solution for $\operatorname{RIEP}(\mathrm{n}-1)$ and $l_{n, n} \neq 0$. Our assertion now follows by induction on $n$.

We conclude this section remarking that a similar discussion can be carried over for complex Hermitian matrices.

\section{The positive semidefinite case}

In view of the discussion of the previous section, it would be interesting to find conditions for the existence of a positive (semi)definite real symmetric solution for $\operatorname{RIEP}(n)$. Clearly, a necessary condition is nonnegativity of the numbers $s_{i}$ whenever $r_{i} \neq 0$ or $l_{i} \neq 0, i=1, \ldots, n$. Nevertheless, this condition is not sufficient even if a real symmetric solution exists, as is demonstrated by the following example. 
Example 34 Let

$$
s_{1}=1, \quad s_{2}=3, \quad s_{3}=5,
$$

and let

$$
l_{1}=r_{1}=[1], \quad l_{2}=r_{2}=\left[\begin{array}{l}
1 \\
1
\end{array}\right], \quad l_{3}=r_{3}=\left[\begin{array}{l}
1 \\
1 \\
1
\end{array}\right] .
$$

The unique solution for $\operatorname{RIEP}(3)$ is the symmetric matrix

$$
\left[\begin{array}{lll}
1 & 2 & 2 \\
2 & 1 & 2 \\
2 & 2 & 1
\end{array}\right]
$$

which is not positive semidefinite.

The following necessary and sufficient condition follows immediately from Theorem 4.

Theorem 35 Let $n \geq 2$ and assume that (20) holds. Assume, further, that $r_{i, i} \neq 0, i=1, \ldots, n$. Then the unique solution for $\operatorname{RIEP}(n)$ is positive semidefinite [positive definite] if and only if $S_{n} \circ\left(R_{n}^{T} R_{n}\right)$ is positive semidefinite [positive definite].

Remark 36 By Theorem 33, in the case that $r_{i, i}=0$ for some $i$ we do not have uniqueness of symmetric solutions for $\operatorname{RIEP}(n)$. Hence, if there exists a symmetric solution for $\operatorname{RIEP}(n)$ then there exist at least two different such solutions $A$ and $B$. Note that $A+c(B-A)$ also forms a symmetric solution for $\operatorname{RIEP}(n)$ for every real number $c$. It thus follows that in this a case it is impossible to have all solutions for $\operatorname{RIEP}(n)$ positive semidefinite. Therefore, in this case we are looking for conditions for the existence of some positive semidefinite solution for $\operatorname{RIEP}(n)$.

The following necessary condition follows immediately from Proposition 3 .

Theorem 37 Let $n \geq 2$ and assume that (27) holds. If there exists a positive semidefinite real symmetric solution for $\operatorname{RIEP}(n)$ then $S_{n} \circ\left(R_{n}^{T} R_{n}\right)$ is positive semidefinite. 
In order to find sufficient conditions for the existence of a positive semidefinite solution for $\operatorname{RIEP}(n)$, we denote by $\sigma(A)$ the least eigenvalue of a real symmetric matrix $A$.

Lemma 38 Let $n \geq 2$ and assume that (27) holds. Assume that there exists a symmetric solution $A$ for $\operatorname{RIEP}(n)$ such that $\sigma(A\langle n-1\rangle)>s_{n}$. If $r_{n} \neq 0$ then $\sigma(A)=s_{n}$.

Proof. Since $r_{n} \neq 0$ it follows that $s_{n}$ is an eigenvalue of $A$. Assume that $\sigma(A) \neq s_{n}$. It follows that $A$ has at least two eigenvalues smaller than or equal to $s_{n}$. By the Cauchy Interlacing Theorem for Hermitian matrices, e.g. [9, Theorem 4.3.8, p. 185], it follows that $\sigma(A\langle\mathrm{n}-1\rangle) \leq s_{n}$, which is a contradiction. Therefore, we have $\sigma(A)=s_{n}$.

Corollary 39 Let $n \geq 2$ and assume that (27) holds. If $r_{i} \neq 0$ for all $i$, $i=1, \ldots, n$, and if $s_{1}>s_{2}>\ldots>s_{n} \geq 0$ then every real symmetric solution $A$ for $\operatorname{RIEP}(n)$ is positive semidefinite. If $s_{n}>0$ then every real symmetric solution for $\operatorname{RIEP}(n)$ is positive definite.

Proof. Note that $A\langle 1\rangle)=\left[s_{1}\right]$ and so $\sigma(A\langle 1\rangle)=s_{1}$. Using Lemma 38 repeatedly we finally obtain $\sigma(A)=s_{n}$, implying our claim.

Remark 40 In view of Remark 36, it follows from Corollary 39 that if $r_{i} \neq 0$ for all $i$ and if $s_{1}>s_{2}>\ldots>s_{n} \geq 0$ then $r_{i, i} \neq 0, i=1, \ldots, n$, and so $\operatorname{RIEP}(n)$ has a unique (positive semidefinite) solution.

The converse of Corollary 39 is, in general, not true. That is, even if every real symmetric solution for $\operatorname{RIEP}(n)$ is positive semidefinite we do not necessarily have $s_{1}>s_{2}>\ldots>s_{n} \geq 0$, as is demonstrated by the following example.

Example 41 Let

$$
s_{1}=2, \quad s_{2}=3,
$$

and let

$$
l_{1}=r_{1}=[1], \quad l_{2}=r_{2}=\left[\begin{array}{l}
1 \\
1
\end{array}\right] .
$$

The unique solution for $\operatorname{RIEP}(2)$ is the positive definite matrix

$$
A=\left[\begin{array}{ll}
2 & 1 \\
1 & 2
\end{array}\right] .
$$

Nevertheless, we do not have $s_{1} \geq s_{2}$. 
We conclude this section with a conjecture motivated by Theorems 35 and 37. One direction of the conjecture is proven in Theorem 37.

Conjecture 42 Let $n \geq 2$, let (27) hold and assume that a solution for $\operatorname{RIEP}(n)$ exists. Then there exists a positive semidefinite [positive definite] real symmetric solution for $\operatorname{RIEP}(n)$ if and only if $S_{n} \circ\left(R_{n}^{T} R_{n}\right)$ is positive semidefinite [positive definite].

In Conjecture 42, the requirement that a solution for $\operatorname{RIEP}(n)$ exists is necessary, as is demonstrated by the following example.

Example 43 Let

$$
s_{1}=2, \quad s_{2}=1
$$

and let

$$
l_{1}=r_{1}=[1], \quad l_{2}=r_{2}=\left[\begin{array}{l}
1 \\
0
\end{array}\right] \text {. }
$$

The unique solution for $\operatorname{RIEP}(1)$ is the matrix $B=[2]$, and so by Theorem 2 there exists no solution for $\operatorname{RIEP}(2)$. Nevertheless, the matrix $S_{2} \circ\left(R_{2}^{T} R_{2}\right)$ is the positive semidefinite matrix

$$
\left[\begin{array}{ll}
2 & 1 \\
1 & 1
\end{array}\right]
$$

\section{The Stieltjes matrix case}

In this section we combine the results of the previous two sections to obtain analogous results for Stieltjes matrices, that is, symmetric $M$-matrices.

The following theorem follows immediately from Theorems 30 and 25 .

Theorem 44 Let $n \geq 2$. There exists a symmetric Z-matrix solution for $\operatorname{RIEP}(n)$ if and only if there exists a symmetric $Z$-matrix solution $B$ for $\operatorname{RIEP}(n-1)$ satisfying

$$
\left\{\begin{array}{l}
\frac{s_{n} \tilde{r}_{n}}{r_{n, n}} \leq \frac{B \tilde{r}_{n}}{r_{n, n}}, \quad r_{n, n} \neq 0 \\
s_{n} \tilde{r}_{n}=B \tilde{r}_{n}, \quad r_{n, n}=0
\end{array},\right.
$$




$$
\left\{\begin{array}{l}
\frac{\tilde{l}_{n}^{T} s_{n}}{l_{n, n}} \leq \frac{\tilde{l}_{n}^{T} B}{l_{n, n}}, \quad l_{n, n} \neq 0 \\
\tilde{l}_{n}^{T} s_{n}=\tilde{l}_{n}^{T} B, \quad l_{n, n}=0
\end{array},\right.
$$

and

$$
l_{n, n} r_{n, n} \neq 0 \quad \Longrightarrow \quad\left(s_{n} I_{n-1}-B\right)\left(\frac{\tilde{l}_{n}}{l_{n, n}}-\frac{\tilde{r}_{n}}{r_{n, n}}\right)=0 .
$$

Furthermore, if $l_{n}$ or $r_{n}$ is positive then a symmetric Z-matrix solution for $\operatorname{RIEP}(n)$ is a Stieltjes matrix if and only if $s_{n} \geq 0$.

Corollary 45 Let $n \geq 2$, and assume that the vectors $l_{i}, i=1, \ldots, n$ are all positive and that (27) holds. There exists a symmetric Z-matrix solution A for $\operatorname{RIEP}(n)$ if and only if there exists a symmetric $Z$-matrix solution $B$ for $\operatorname{RIEP}(n-1)$ satisfying $s_{n} \tilde{r}_{n} \leq B \tilde{r}_{n}$. The solution $A$ is a Stieltjes matrix if and only if $s_{n} \geq 0$.

The following nonrecursive sufficient condition from Theorem 27.

Theorem 46 Let $n \geq 2$, and assume that the vectors $l_{i}, i=1, \ldots, n$ are all positive, that (27) holds, and that the numbers $s_{1}, \ldots, s_{n}$ are all positive. If we have

$$
s_{i} M_{i}^{r} \leq s_{i-1} m_{i}^{r}, \quad i=2, \ldots, n
$$

then there exists a (unique) Stieltjes matrix solution $A$ for $\operatorname{RIEP}(n)$.

Proof. By Theorem 27 there exists a unique $M$-matrix solution $A$ for $\operatorname{RIEP}(n)$. Since $A^{T}$ also solves the problem, it follows that $A=A^{T}$ and the result follows.

\section{The inverse $M$-matrix case}

It is well known that for a nonsingular $M$-matrix $A$ we have $A^{-1} \geq 0$. Accordingly, a nonnegative matrix $A$ is called inverse $M$-matrix if it is invertible and $A^{-1}$ is an $M$-matrix. An overview of characterizations of nonnegative matrices that are inverse $M$-matrices can be found in [10].In this section we discuss, as a final special case, inverse $M$-matrix solutions for $\operatorname{RIEP}(n)$.

The following theorem follows immediately from two results of [10]. 
Theorem 47 Let $A \in \mathbb{R}^{n, n}$ be partitioned as in (3). Then $A$ is an inverse $M$-matrix if and only if $B$ is an inverse $M$-matrix and

$$
\begin{gathered}
v=B^{-1} y \geq 0, \\
u^{T}=x^{T} B^{-1} \geq 0, \\
s=z-u^{T} B v>0,
\end{gathered}
$$

and

$$
v u^{T} \leq-s B^{-1}, \quad \text { except for the diagonal entries. }
$$

Proof. By Corollary 3 in [10], if $A$ is an inverse $M$-matrix then $B$ is an inverse $M$-matrix. By Theorem 8 in [10], if $B$ is an inverse $M$-matrix then $A$ is an inverse $M$-matrix if and only if (28)-(31) hold. Our claim follows.

The next result gives necessary and sufficient recursive conditions for the existence of an inverse $M$-matrix solution for $\operatorname{RIEP}(n)$.

Theorem 48 Let $n \geq 2$. There exists an inverse $M$-matrix solution for $\operatorname{RIEP}(n)$ if and only if $s_{n}>0$ and there exists an inverse $M$-matrix solution $B$ for $\operatorname{RIEP}(n-1)$ satisfying

$$
\begin{gathered}
\left\{\begin{array}{l}
\frac{N \tilde{r}_{n}}{r_{n, n}} \geq 0, \quad r_{n, n} \neq 0 \\
N \tilde{r}_{n}=0, \quad r_{n, n}=0
\end{array}\right. \\
\begin{cases}\frac{\tilde{l}_{n}^{T} N}{l_{n, n}} \geq 0, & l_{n, n} \neq 0 \\
\tilde{l}_{n}^{T} N=0, & l_{n, n}=0\end{cases} \\
l_{n, n} r_{n, n} \neq 0 \Longrightarrow \frac{\tilde{l}_{n}^{T} N \tilde{r}_{n}}{l_{n, n} r_{n, n}}<1,
\end{gathered}
$$

and, except for the diagonal entries,

$$
l_{n, n} r_{n, n} \neq 0 \Rightarrow s_{n}\left(\frac{\tilde{l}_{n}^{T} N \tilde{r}_{n}}{l_{n, n} r_{n, n}}-1\right) B^{-1} \geq \frac{N \tilde{r}_{n} \tilde{l}_{n}^{T} N}{l_{n, n} r_{n, n}}
$$

where $N=s_{n} B^{-1}-I_{n-1}$. 
Proof. As in the proof of Theorem 2, partition $A$ as in (3). If $A$ is an inverse $M$-matrix solution for $\operatorname{RIEP}(n)$ then, as is well known, its eigenvalues lie in the open right half plane, and so the real eigenvalue $s_{n}$ must be positive. Furthermore, by Theorem $47, B$ is an inverse $M$-matrix and (28)-(31) hold. Finally, we have (4)-(7). Distinguish between four cases:

1. $r_{n, n}=0, l_{n, n} \neq 0$. Here $x$ is given by (8), and so it follows from (29) that $\frac{\tilde{i}_{n}^{T} N}{l_{n, n}} \geq 0$. By Theorem 2 we have $B \tilde{r}_{n}=s_{n} \tilde{r}_{n}$, implying that $N \tilde{r}_{n}=0$.

2. $l_{n, n}=0, r_{n, n} \neq 0$. Here $y$ is given by (9), and so it follows from (28) that $\frac{N \tilde{r}_{n}}{r_{n, n}} \geq 0$. By Theorem 2 we have $\tilde{l}_{n}^{T} N=0$.

3. $l_{n, n}=r_{n, n}=0$. Similarly to the previous cases prove that $N \tilde{r}_{n}=0$ and $\tilde{l}_{n}^{T} N=0$.

4. $l_{n, n} \neq 0, r_{n, n} \neq 0$. Here $x$ is given by (8), $y$ is given by (9), and $z$ is given by (10). It follows from (28) that $\frac{N \tilde{r}_{n}}{r_{n, n}} \geq 0$, and from (29) that $\frac{\tilde{l}_{n}^{T} N}{l_{n, n}} \geq 0$. It follows from (30) that

$$
\begin{gathered}
s=z-u^{T} B v \\
=s_{n}-\frac{\tilde{l}_{n}^{T}\left(s_{n} I_{n-1}-B\right) \tilde{r}_{n}}{l_{n, n} r_{n, n}}-\frac{\tilde{l}_{n}^{T}\left(s_{n} I_{n-1}-B\right)}{l_{n, n}} B^{-1} B B^{-1} \frac{\left(s_{n} I_{n-1}-B\right) \tilde{r}_{n}}{r_{n, n}} \\
=s_{n}\left(1-\frac{\tilde{l}_{n}^{T} N \tilde{r}_{n}}{l_{n, n} r_{n, n}}\right)>0 .
\end{gathered}
$$

Since $s_{n}>0$, it now follows that $\frac{\tilde{l}_{n}^{T} N \tilde{r}_{n}}{l_{n, n} r_{n, n}}<1$. Finally, it follows from (31) that, except for the diagonal entries,

$$
\begin{aligned}
\frac{N \tilde{r}_{n} \tilde{l}_{n}^{T} N}{l_{n, n} r_{n, n}}= & B^{-1} \frac{\left(s_{n} I_{n-1}-B\right) \tilde{r}_{n}}{r_{n, n}} \frac{\tilde{l}_{n}^{T}\left(s_{n} I_{n-1}-B\right)}{l_{n, n}} B^{-1}=v u^{T} \\
& \leq-s B^{-1}=s_{n}\left(\frac{\tilde{l}_{n}^{T} N \tilde{r}_{n}}{l_{n, n} r_{n, n}}-1\right) B^{-1} .
\end{aligned}
$$


We have thus proven that if $A$ is an inverse $M$-matrix solution for $\operatorname{RIEP}(n)$

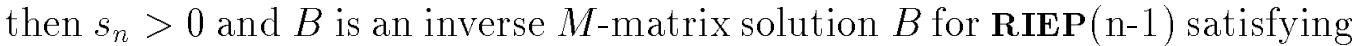
$(32)-(35)$.

Conversely, assume that $s_{n}>0$ and $B$ is an inverse $M$-matrix solution $B$ for $\operatorname{RIEP}(\mathrm{n}-1)$ satisfying $(32)-(35)$. We show that $x, y$ and $z$ can be chosen such that (28)-(31) hold, and so by Theorem $47, A$ is an inverse $M$-matrix. Here too we distinguish between four cases:

1. $r_{n, n}=0, l_{n, n} \neq 0$. Here $x$ is given by (8), and by (33) we obtain (29). Note that $y$ can be chosen arbitrarily, and and $z$ should be chosen such that (7) holds. If we choose $y=0$ then we obtain (28) and $z=s_{n}$. It follows that $z-u^{T} B v=s_{n}>0$, and so we also have (30). Finally, since $v=0$, since $s>0$ and since $B^{-1}$ is an $M$-matrix, it follows that (31) holds (except for the diagonal entries).

2. $l_{n, n}=0, r_{n, n} \neq 0$. Here $y$ is given by (9), and by (32) we obtain (28). The vector $x$ can be chosen arbitrarily, so we choose $x=0$. The proof follows as in the previous case.

3. $l_{n, n}=r_{n, n}=0$. Here $x$ and $y$ should be chosen such that $x^{T} \tilde{r}_{n}=\tilde{l}_{n}^{T} y=$ 0 and $z$ can be chosen arbitrarily. We choose $x=y=0$ and the proof follows.

4. $l_{n, n} \neq 0, r_{n, n} \neq 0$. Here $x$ is given by (8), $y$ is given by (9), and $z$ is given by (10). By (32) and (33) we obtain (28) and (29) respectively. Finally, similarly to the corresponding case in the proof of the other direction, (34) implies (30) and (35) implies (31).

Note that Conditions (32)-(33) imply immediately Conditions (16)-(17) by multiplying the inequality by the nonnegative matrix $B$. This is not surprising, since an inverse $M$-matrix is a nonnegative matrix. The converse, however, does not hold in general. The following example shows that although (16)-(17) is satisfied, (32)-(33) do not hold.

Example 49 Let

$$
s_{1}=2, \quad s_{2}=5.2361, \quad s_{3}=21.2552,
$$


and let

$$
l_{1}=r_{1}=[1], \quad l_{2}=r_{2}=\left[\begin{array}{l}
0.5257 \\
0.8507
\end{array}\right], \quad l_{3}=r_{3}=\left[\begin{array}{l}
0.1349 \\
0.3859 \\
0.9126
\end{array}\right] .
$$

The unique solution for $\operatorname{RIEP}(3)$ is the nonnegative matrix

$$
A=\left[\begin{array}{ccc}
2 & 2 & 2 \\
2 & 4 & 7 \\
2 & 7 & 18
\end{array}\right]
$$

which is not an inverse $M$-matrix since

$$
A^{-1}=\left[\begin{array}{rrr}
1.6429 & -1.5714 & 0.4286 \\
-1.5714 & 2.2857 & -0.7143 \\
0.4286 & -0.7143 & 0.2857
\end{array}\right]
$$

Indeed, the unique nonnegative solution $B=\left[\begin{array}{ll}2 & 2 \\ 2 & 4\end{array}\right]$ for $\operatorname{RIEP}(2)$ satisfies (16), as

$$
s_{3} \tilde{r}_{3}=\left[\begin{array}{l}
2.8673 \\
8.2024
\end{array}\right] \geq\left[\begin{array}{l}
1.0416 \\
1.8134
\end{array}\right]=B \tilde{r}_{3} .
$$

However, $B$ does not satisfy (32), since the vector

$$
N \tilde{r}_{3}=\left(s_{3} B^{-1}-I_{2}\right) \tilde{r}_{3}=\left[\begin{array}{r}
-1.3688 \\
2.2816
\end{array}\right]
$$

is not nonnegative.

\section{Summary}

We have given a detailed analysis of the recursive inverse eigenvalue problem, providing recursive and nonrecursive existence and uniqueness results for general matrices as well as specific classes of matrices. We summarize the results in the following table. 


\section{Table of results:}

\begin{tabular}{|c|c|c|c|c|}
\hline Result & class of matrices & existence & uniqueness & recurs./nonrec. \\
\hline Theorem 2 & $F^{n, n}$ & $*$ & $*$ & recursive \\
\hline Theorem 4 & $F^{n, n}$ & $*$ & $*$ & nonrecursive \\
\hline Theorem 6 & nonneg. matrices & * & & recursive \\
\hline Theorem 6 & pos. matrices & $*$ & & recursive \\
\hline Corollary 17 & nonneg. matrices & * & $*$ & recursive \\
\hline Corollary 17 & pos. matrices & $*$ & $*$ & recursive \\
\hline Corollary 22 & nonneg. matrices & & $*$ & recursive \\
\hline Corollary 23 & nonneg. matrices & & $*$ & recursive \\
\hline Theorem 25 & Z-matrices & $*$ & & recursive \\
\hline Theorem 25 & M-matrices & $*$ & & recursive \\
\hline Theorem 26 & Z-matrices & $*$ & & nonrecursive \\
\hline Theorem 27 & M-matrices & $*$ & $*$ & recursive \\
\hline Theorem 28 & M-matrices & & * & recursive \\
\hline Theorem 30 & real symm. matrices & $*$ & & recursive \\
\hline Theorem 33 & real symm. matrices & $*$ & $*$ & nonrecursive \\
\hline Theorem 35 & pos. semidef. matrices & $*$ & $*$ & nonrecursive \\
\hline Corollary 39 & pos. semidef. matrices & $*$ & & nonrecursive \\
\hline Theorem 43 & Stieltjes matrices & $*$ & $*$ & recursive \\
\hline Corollary 44 & Stieltjes matrices & $*$ & & recursive \\
\hline Theorem 45 & Stieltjes matrices & $*$ & $*$ & recursive \\
\hline Theorem 47 & inverse M-matrices & $*$ & & recursive \\
\hline
\end{tabular}

\section{References}

[1] A. Ben-Israel and T.N.E. Greville, Generalized Matrix Inverses: Theory and Applications, John Wiley, New York, 1974.

[2] A. Berman and R. Plemmons, Nonnegative Matrices in Mathematical Sciences, SIAM, Philadelphia, 1994.

[3] D. Boley and G.H. Golub, A survey of matrix inverse eigenvalue problems, Inverse problems 3 (1987), 595-622.

[4] M. Boyle and D. Handelman, The spectra of non-negative matrices via symbolic dynamics, Ann. Math. 133 (1991), 249-316. 
[5] M.T. Chu, Inverse eigenvalue problems, SIAM Rev. 40 (1998), no. 1, 1-39

[6] S. Friedland, On an inverse problem for nonnegative and eventually nonnegative matrices, Israel J. Math. 29 (1978), 43-60.

[7] G. Frobenius, Über Matrizen aus positiven Elementen, S.-B. Preuss. Akad. Wiss. (1909), 471-476.

[8] H. Hochstadt, On some inverse problems in matrix theory, Arch. Math. 18 (1967), 201-207.

[9] R.A. Horn and C.R. Johnson, Matrix Analysis, Cambridge University Press, Cambridge, 1985.

[10] C.R. Johnson, Inverse M-matrices, Lin. Alg. Appl. 47 (1982), 195-216.

[11] D.M. Koteljanskii, On some properties of matrices with positive elements, Transl. Amer. Math. Soc. Ser. 227 (1963), 9-18.

[12] T.J. Laffey, Inverse eigenvalue problems for matrices, Proc. Royal Irish. Acad. 95 A (suppl.) (1995), 81-88.

[13] R. Loewy and D. London, A note on an inverse problem for nonnegative matrices, Lin. and Multilin. Alg. 6 (1978), 83-90.

[14] G.N. de Oliveira, Note on an inverse characteristic value problem, $\mathrm{Nu}$ mer. Math. 15 (1970), 345-347. 